\title{
Performance Analysis of Node Mobility in Beacon and Non-Beacon enabled IEEE 802.15 .4 based Wireless Sensor Network
}

\author{
Surender.R \\ Department of Electronics Engineering \\ School of Engineering and Technology \\ Pondicherry University, \\ Pondicherry, India
}

\author{
P. Samundiswary \\ Department of Electronics Engineering \\ School of Engineering and Technology \\ Pondicherry University \\ Pondicherry, India
}

\begin{abstract}
Recent advances in wireless communication technologies have enabled the development of Wireless Sensor Networks (WSNs) that consist of low cost, power and small-size sensor nodes. ZigBee, an IEEE 802.15.4 based wireless sensor networks have been the promise of facilitating large-scale and real-time data processing in complex environments. Mobility management is a challenging task in the IEEE 802.15.4 based wireless sensor network. Work in this paper comprises of recovery of the network in case of PAN coordinator failure using mobile node with trajectory. The simulation model consists of IEEE 802.15.4 based wireless sensor network which involves a mobile node deployed near PAN coordinator. The deployed mobile node routes through the coverage area of each PAN coordinator to recover the network. The work is also implicated with Beacon and Nonbeacon enabled IEEE 802.15.4 based wireless sensor network. The simulation model is done by using OPNET. Performances metrics like throughput and delay in the network are determined and analyzed.
\end{abstract}

\section{Keywords}

ZigBee, IEEE 802.15.4, mobile node, trajectory, PAN coordinator

\section{INTRODUCTION}

Rapid development of low-rate Wireless Personal Area Networks (LR-WPANs) technology has attracted a lot of attention due to its huge application space. LR-WPANs adopt IEEE 802.15.4 as a communication standard for Zigbee networks. IEEE 802.15.4 based WSN standard has also gained significant attention among researchers in recent years. The IEEE 802.15.4 standard [1] is used in the application space of industrial automation, intelligent measurement, intelligent building and remote medical care because of its low level of complexity, low cost and low power consumption. Zigbee technology aims at remote control and sensor applications, which is suitable for operation in harsh radio environments and isolated locations. IEEE 802.15.4 based WSN defines the physical and MAC layers [2].

The MAC layer of IEEE 802.15.4 standards operates in two different types of modes. They are beacon enabled and nonbeacon enabled mode. Periodic transmission of beacon messages are the features of beacon enabled mode for network association and synchronization [3]. Beacon enabled can operate network-wide to maintain synchronization and allows the mode to operate on slotted Carrier Sense Multiple Access with Collision Avoidance (CSMA/CA) mechanism. On other hand, non-beacon enabled mode nodes are not synchronized, due to the absence of periodic beacon transmissions. Therefore, the decentralised communication among the nodes in this mode is facilitated by unslotted CSMA/CA mechanism [4].

Apart from the absence of periodic beacons and its consequences (e.g., absence of network-wide synchronization and superframe structure), their nature of time evolution is the major difference between slotted and unslotted CSMA/CA mechanisms. Several studies have investigated energy consumption in non-beacon enabled Zigbee WSNs [5]. A comprehensive performance evaluation of Zigbee wireless networks in beacon enabled and non-beacon enabled modes has been performed by Bilgin et al. [6]. Their results showed that the utility of either beacon enabled or non-beacon enabled mode is dependent on the specific application. However the analysis of the node mobility deployed near PAN coordinator is not examined to improve the performance in case of coordinator failure for either beacon enabled or non-beacon enabled network. An attempt has been made to implement mobile node in adjacent with the PAN coordinator for both beacon enabled and non- beacon enabled network and it is exploited in this paper.

The paper is structured as follows: Section 2 presents the comprehensive view of IEEE 802.15.4 standard. Section 3 gives an overview of proposed network model in the network. Simulated results and discussion are presented in Section 4. Section 5 discusses about conclusion and future work.

\section{OVERVIEW OF IEEE 802.15.4 ARCHITECTURE}

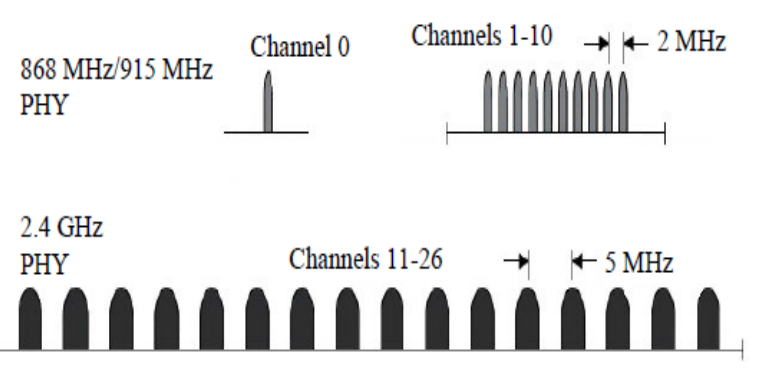


Fig. 1 illustrates various frequency bands of Zigbee

IEEE 802.154 based WSN is the only wireless standards technology designed to address the unique needs of low-cost, low-power wireless sensor and control networks. Fig. 1 shows the frequency bands of ZigBee standards. The device employed in this network has maximum operating at $2.4 \mathrm{GHz}$ frequency with data rate of $240 \mathrm{Kbps}$ [7]. IEEE 802.15.4 based WSN needs miniature power to operate and has the opportunity for growth into new markets. ZigBee specification establishes the framework for the Network and Application layers based on the PHY and MAC layers specified by IEEE 802.15.4 standard [8]. Two types of devices are specified for IEEE 802.15.4 framework. They are Full Function Device (FFD) and Reduced Function Device (RFD). An example for FFD is PAN Coordinator and can also functions like router. It has more responsibility to maintain routing tables, participate in route discovery and repair, maintain beaconing framework. It has also handles the node that joins the network. An end device is RFD. It has the capability of communicating with any other devices within its transmission range. On the other hand, RFD simply maintains the minimum amount of knowledge to stay on the network, and it does not participate in routing.

Specifically, IEEE 802.15.4 controls the access to radio channel using the unslotted CSMA/CA or the optional slotted CSMA/CA mechanism, as respectively utilized by the either beaconless and beaconed modes at the MAC layer. In nonbeacon-enabled networks, an unslotted CSMA/CA channel access mechanism is used. ZigBee routers typically have their receivers continuously active, requiring a more robust power supply in this type of network. However, this allows for heterogeneous networks in which some devices receive continuously, while others only transmit when an external stimulus is detected.

In beacon-enabled networks, special nodes called ZigBee routers transmit periodic beacons to confirm their presence to other network node [9]. Nodes may sleep between beacons, thus lowering their duty cycle and extending their battery life. Beacon intervals depend on data rate. However, low duty cycle operation with long beacon intervals requires precise timing, which can conflict with the need for low product cost. In beacon enabled networks, nodes only need to be active while a beacon is being transmitted. In non-beacon-enabled networks, power consumption is asymmetrical some devices are always active, while others spend most of their time sleeping long battery life.

Several proposals have been made to study mobility effects in WSNs using ZigBee standard. Francisco J. Claudios et al. [10] has focused on mobility mechanisms for creation and reconfiguration of cluster tree networks based on IEEE 802.15.4. Taehong Kim et al [11] has proposed an application to support seamless communication and a high data reception rate. In this approach, mobile node connectivity with PAN coordinator has been proposed to improve the performance of the network. The main contribution of this work is to analyze the performance of mobility in the network.

\section{PROPOSED NETWORK MODEL}

The type of mobility model to be chosen for wireless sensor networks depends on the nature of application. It's hard s to produce a standard definition for mobility because there are different categories of mobility according to different principles. Based on the movement scope, mobility can be classified into two types.
- Device which moves internally in its networks are Intramobility

- and device moving between networks are said to be Intermobility.

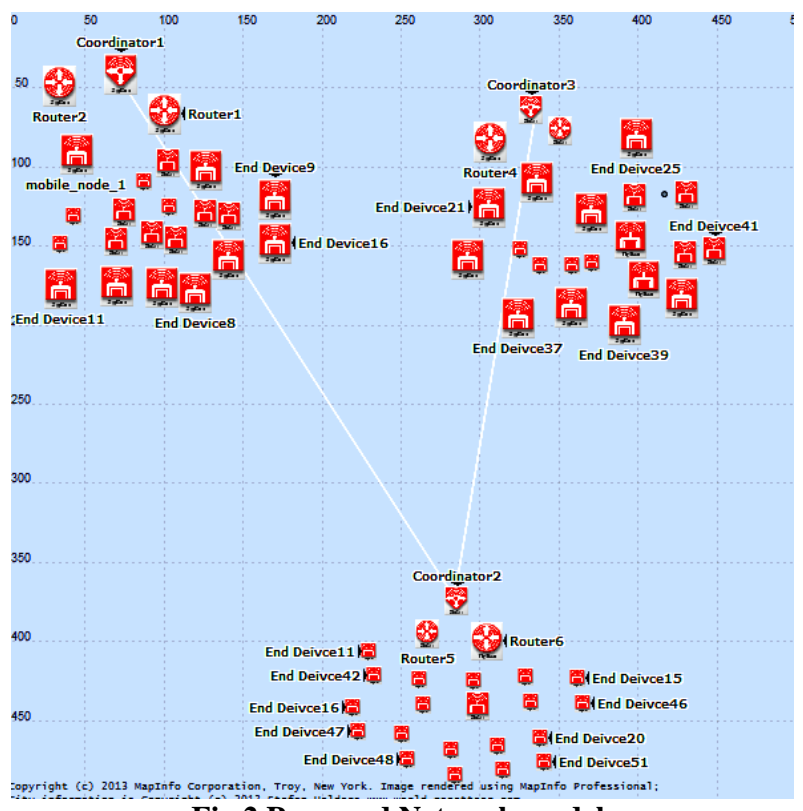

Fig.2 Proposed Network model

Three kinds of mobility are classified based on the device type. They are.

- If the sensor node itself is a moving object, generating data and delivering the data to a sink, then this type is defined as Sensor-mobility.

- If the mobile sink node is used to gather information from sensors by visiting them and forward the sensing data to the user, then this type of mobility is called Sink-mobility.

- If the users communicates with the nodes of sensor networks and receive data from sink through multi-hop communications, then that type is called User-mobility [12].

Fig. 2 illustrates the proposed network model which uses the intra- mobility model of IEEE 802.15.4 based WSN. The model is configured by three groups of PAN coordinator, router and end devices. Each PAN coordinator is deployed with router to establish connection with end devices. When failure of PAN is encountered, mobile node establishes connection with the help of trajectory path to the next PAN.

The proposed network contains three PANs named as PAN ID 1,2 , and 3 . The network also contains a mobile node, initially placed next to PAN 1, with its PAN ID set to auto-assigned. The three PANs have their transmit power configured to 2 $\mathrm{mW}$ so that their coverage areas do not overlap. The trajectory of mobile node is configured to take that node through the coverage area of each of the three PANs. Based on this trajectory, it is expected that mobile node will initially join PAN 1, then switch to PAN 2, and finally to PAN 3. The traffic on each node except mobile node is configured as random destination. When the mobile node establishes connection with network, they will select a random node within their own PAN and drive traffic to that node. Mobile node is configured to send traffic to that particular PAN node. 


\section{SIMULATION RESULTS}

The main objective of this paper is to analyze node mobility deployed near PAN coordinator in IEEE 802.15.4 based wireless sensor network working with beacon and non beacon mode considering $500 \times 500 \mathrm{~m}^{2}$ for 100 nodes. The OPNET attributes used in the simulation are tabulated in table1.

Table 1 Opnet Attributes For Simulation

\begin{tabular}{|l|c|}
\hline Parameters & Values \\
\hline Zigbee Frequency band & $2.4 \mathrm{GHz}$ \\
\hline Data rate & $240 \mathrm{Kbps}$ \\
\hline Protocol & AODV \\
\hline Coverage area & $500 \times 500 \mathrm{~m}^{2}$ \\
\hline No. of nodes & 100 \\
\hline Topology & Cluster Tree \\
\hline Network & Beacon and Non Beacon \\
& enabled \\
\hline ACK wait duration & $5 \mathrm{~ms}$ \\
\hline Transmission power & $2 \mathrm{~mW}$ \\
\hline
\end{tabular}

\subsection{Average throughput analysis}

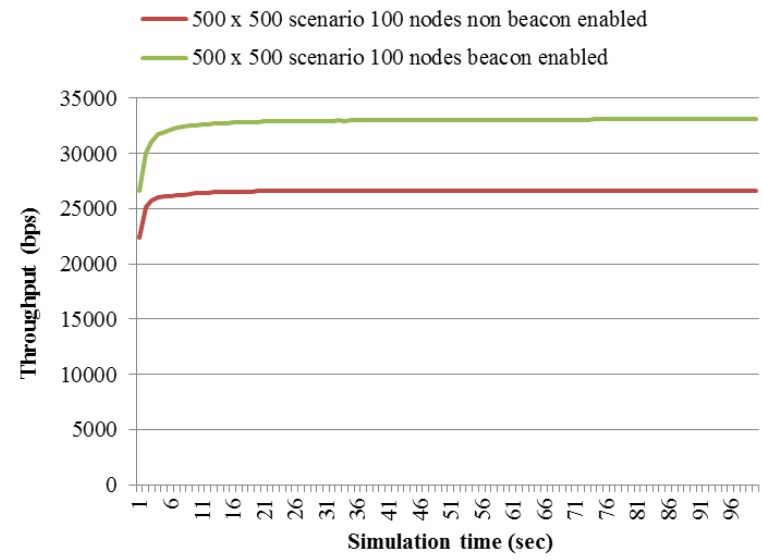

Fig 3 Comparison of average throughput for beacon and non- beacon enabled scenario for 100 nodes

The simulation result shown in fig 3 compares average throughput of beacon enabled and non- beacon enabled IEEE 802.15 .4 based WSN for $500 \times 500 \mathrm{~m}^{2}$ scenario with 100 nodes. The throughput of the network for beacon and non -beacon mode is found to be $33 \mathrm{Kbps}$ and $27 \mathrm{Kbps}$ respectively. The beacon enabled network shows better throughput because of periodic function of the beacon enabled device. The beacon order and super frame order are used to communicate with each of the devices in network through the trajectory and with other PAN through the mobile node deployed in the network. In non-beacon enabled network, devices directly come into action so as no ACK mechanism is involved. As a result more packets may be dropped in the network which in turn reduces the throughput. This characteristic feature makes beacon enabled network more effective than that of non-beacon enabled network.

\subsection{Average MAC delay analysis}

The simulation result shown in fig 4 depicts the average MAC delay of beacon enabled and non- beacon enabled network for coverage area of $500 \times 500 \mathrm{~m}^{2}$ with 100nodes. Generally average MAC delay refers to time taken by the devices to access the channel for connection. From the simulation result, it is observed that the MAC delay of beacon and non -beacon mode is found to be $3.2 \mathrm{~ms}$ and $2.9 \mathrm{~ms}$ correspondingly. As beacon enabled network involves ACK mechanism, delay is high. It waits for acknowledgement and it establishes communication with devices involved in the network after receiving the acknowledgement. In non beacon enabled network, a node directly comes into action without the involvement of ACK mechanism in the network. This characteristic feature makes delay to be low for non-beacon enabled network.

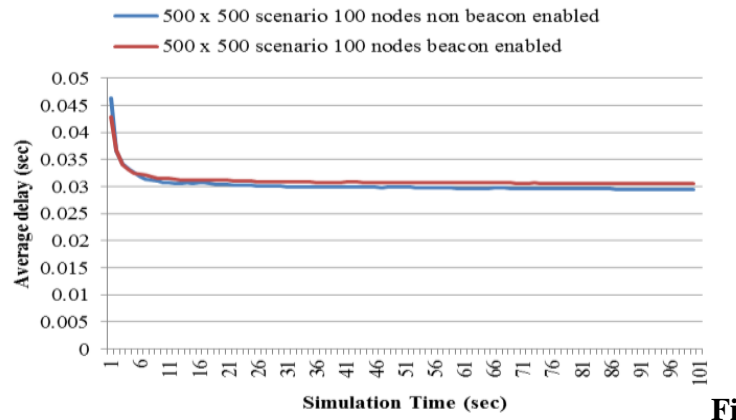

4 Comparison of average MAC delay for beacon and nonbeacon enabled scenario 100 nodes

\subsection{Average end to end delay analysis}

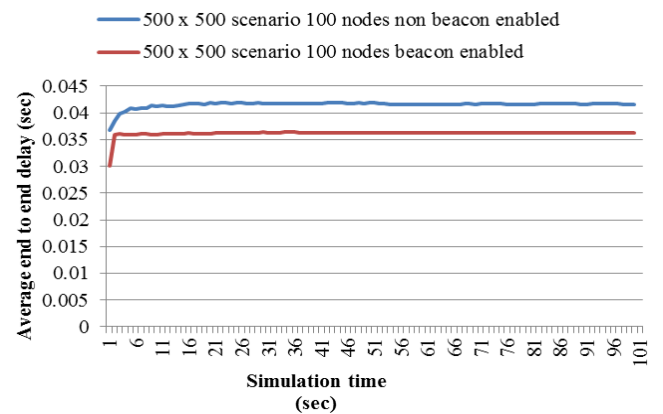

Fig 5 Comparison of average end to end delay of beacon and non- beacon enabled scenario for 100 nodes

End to end delay refers to consolidation of overall delay like queuing and MAC delay which are involved in the network. The simulation result shown in fig 5 portrays average end to end delay for beacon enabled and non- beacon enabled network considering $500 \times 500 \mathrm{~m}^{2}$ as terrain area with 100 nodes. Average end to end delay of beacon and non-beacon enabled network is found to be $4.5 \mathrm{~ms}$ and $3.3 \mathrm{~ms}$ respectively. In beacon enabled network, end to end delay is high because it includes the delay arises due to ACK mechanism in addition to the queuing delay and MAC delay involved in the network. End to end delay is low in non- beacon enabled because nodes directly communicates with the other PAN coordinator through the deployed mobile node and without the usage of ACK mechanism. 


\section{CONCLUSION AND FUTURE SCOPE}

In this paper, performance analysis of node mobility deployed near PAN coordinator in beacon and non beacon enabled IEEE 802.15.4 based wireless sensor network has been investigated to recover the network with the help of mobile node in case of PAN coordinator failure. The IEEE 802.15.4 based WSN is simulated by using OPNET. From the simulation results, it is observed that beacon enabled network outperforms the non-beacon enabled network in terms of throughput due to less packet loss obtained through beacon enabled IEEE 802.15.4 based WSN. However MAC delay and end to end delay is higher for beacon enabled network compared to that of non-beacon enabled IEEE 802.15.4 based WSN. Future work focus on evaluation of performance metrics based on different routing protocol and security algorithms

\section{REFERENCES}

[1] Yong Pan , Ping "Research and Implementation of a Mobility Management Mechanism for Wireless Sensor Networks based on IEEE 802.15.4", Proceedings of Fourth International Conference on Cyber Technology in Automation, Control, and Intelligent Systems, Kunming, Malaysia, pp 260 - 264, March 2011.

[2] Pegatoquet, A., Auguin, M., Jemaa, M.B. “An Efficient Mobility Management Approach for IEEE 802.15.4/ZigBee Nodes", Proceedings of Fourth International Conference on High Performance Computing and Communication, Liverpool, England, pp 897 - 902, June 2012.

[3] Yanjun Zhang, Siye Wang, Zhenyu Liu, Wenbiao Zhou, Dake Liu " Performance Analysis of Wireless Sensor Network Based on NS-2," Proceedings of International Conference on Systems and Informatics, Yantai , China, pp 1445 - 1448, May 2012.

[4] M. Gribaudo, D. Manini, A. Nordio, and C. Chiasserini, "Transient Analysis of IEEE 802.15.4 Sensor Networks", IEEE Transactions on Wireless Communications, Volume 10, Issue 4, pp. 1165 -1175, April 2011.

[5] T.H.Woon and T.C. Wan ," Performance Evaluation of IEEE 802.15.4 Ad Hoc Wireless Sensor Networks: Simulation Approach," Proceedings of IEEE Conference on Systems, Man and Cybernetics, Taipei, Taiwan, pp 1443 - 1448, October 2006.

[6] B.E. Bilgin, V.C. Gungor. "Performance evaluations of zigbee in different smart grid environments", Computer Networks, Istanbul, Turkey, Volume 56, Issue 8, pp 2196-2205,May 2012.
[7] S. Mohanty, S.K. Patra. "Quality of service analysis in IEEE 802.15.4 mesh networks using MANET routing," Proceedings of International Conference on Computing Communication and Networking Technologies, Karur ,Tamil Nadu Pages. 1-7, July 2010

[8] V. Kumar, S. Tiwari. "Performance of routing protocols for beacon-enabled IEEE 802.15.4 WSNs with different duty cycle." Proceedings of International Conference on Devices and Communications, Mesra, Jharkhand, pp. 1-5, 24-25, February 2011.

[9] Pollin, S.; Ergen, M.; Ergen, S.C.; Bougard, B.; Catthoor, F.; Bahai, A.;Varaiya, P.; "Performance Analysis of Slotted Carrier Sense IEEE 802.15.4 Acknowledged Uplink Transmissions",. IEEE Wireless Communications and Networking Conference, Las Vegas, Nevada, pp: 1559 - 1564, April 2008.

[10] F. Claudio, R. Radeke, D. Marandin, P. Todorova, and S. Tomic, "Performance study of reconfiguration algorithms in cluster-tree topologies for wireless sensor networks," Proceedings of IEEE $18^{\text {th }}$ International Symposium on Personal, Indoor and Mobile Radio Communications, Athens, Greece, pp. 1-5, September2007.

[11] Taehong Kim ; Sukbin Seo ; Chong Poh Kit ; Youngsoo Kim, "Experiments on mobile sink application in ZigBee networks", IEEE International Symposium on Consumer Electronics, Vilamoura, Portugal, pp 1 -4 , April 2008

[12] T. Sun, N. Liang, L. Chen, and M. Gerla, "Evaluating mobility support in zigbee networks," Proceedings of international Conference on Embedded and Ubiquitous Computing, Volume 4808, Taipei, Taiwan, pp 87-100, December 2007.

\section{BIBLIOGRAPHY}

R. Surender received his B.Tech Degree from Rajiv Gandhi College of Engineering and Technology affiliated to Pondicherry University, Pondicherry in 2006. He has obtained his M.Tech. Degree from Pondicherry Engineering College affiliated to Pondicherry University, Pondicherry in 2008. He is currently working as Project Fellow in Department of Electronics Engineering of School of Engineering and Technology, Pondicherry University, Pondicherry, India. His areas of interests include Wireless communication, Sensor networks and Computer communication.

P. Samundiswary received the B.Tech. degree (1997), M.Tech. degree (2003) and Ph.D. (2011) in the Department of Electronics and Communication Engineering from Pondicherry Engineering College affiliated to Pondicherry University, India. She has nearly 15 years of teaching experience. She is currently working as Assistant Professor in the Dept. of Electronics Engineering, School of Engineering and Technology, Pondicherry University, Pondicherry, India. Her research interests include Wireless Communication and Wireless Networks. 